Ann. Biol. anim. Bioch. Biophys., I969, 9 (3), 351-360.

\title{
COMPARAISON DE DIFFERENTES TECHNIQUES DE TRANSPLANTATION DES BLASTOCYSTES CHEZ LA LAPINE
}

\author{
J. TESTART \\ avec la collaboration technique de Anne-Marie MÉLIÈres \\ Station centrale de Physiologie animale, \\ Centre national de Recherches zootechniques, 78 -Jouy-en-Josas \\ Institut national de la Recherche agronomique
}

\section{SOMMAIRE}

Le but de ce travail est de rechercher, chez la Lapine, l'influence sur le taux d'implantations et la survie embryonnaire de la technique de transplantation des blastocystes par " inovulation ".

Les stimulations de la vulve ou du vagin, impliquées par cette technique, sont sans effet sur le rendement du transfert.

Seul le passage intracervical du cathéter d'inovulation affecte le rendement; l'expulsion hors de l'utérus des blastocystes ainsi transplantés n'est pas en cause dans ce résultat.

\section{INTRODUCTION}

Le transfert par voie vaginale d'œufs fécondés dans une femelle hôte, ou “inovulation " (BEATTY, I95I), a été réalisé dans quelques espèces (Vache, Chèvre, Lapine, Souris). Le rendement, c'est-à-dire le rapport entre le nombre de fœtus se développant et le nombre d'œufs transplantés, varie d'une expérience à l'autre ; c'est ainsi que, chez la Lapine, deux recherches conduites simultanément aboutissaient aux conclusions suivantes :

Pour HAFEZ (rg62), l'inovulation par cathétérisation transcervicale est aussi efficace que le transfert direct dans l'utérus $(59$ p. Ioo des blastocystes implantés contre 54 à $60 \mathrm{p}$. IOO).

DAUZIER (I962), constate que l'utilisation d'une canule guidant le cathéter jusqu'au cervix entraîne une réduction importante du taux d'implantation : I7 p. Ioo contre 47 p. Ioo si le transfert est pratiqué via le cervix, mais en perforant la paroi vaginale pour éviter le passage du cathéter par la vulve. 
A partir des résultats d'autres expériences chez la Vache (Dowling, I949; LAMMING et RowSON, I952; DzIUK et al., I958; AVERY et al., I962; HAFEZ et Sugie, I963; Rowson, BenNetT et HARPER, I964; MutTter, Graden et Olds, I965 : Sugre, I965; Rowson et Moor, I966) et la Chèvre (Otsuki et Soma, I964), deux causes ont été envisagées pour expliquer les rendements faibles ou inconstants : les contractions utérines déclenchées par stimulation du col et responsables de l'expulsion des œufs dans le vagin ou l'infection résultant du transport, par le cathéter, de matériaux septiques du vagin ou du cervix dans l'utérus en phase lutéale.

Nous avons répété, chez la Lapine, des expériences de transfert dans le but d'éprouver l'influence, lors du passage à travers le col ou la vulve, des stimulations nerveuses ou d'autres facteurs.

\section{MATÉRIEL E'T MÉTHODES}

Cinquante-quatre lapines Grand Russe et Néo-Zélandaise âgées de 4,5 à 6,5 mois ont reçu, 4 jours après coït avec un mâle vasectomisé, des blastocystes de 4 jours suivant les modalités de transfert figurant au tableau I (Exp. I). Quatre à 7 blastocystes étaient déposés dans chaque corne utérine, $\mathrm{a} 3 \mathrm{~cm}$ du cervix. Les lapines receveuses étaient anesthésiées au thiopental.

Les blastocystes de 4 jours des lapines donneuses étaient récupérés par perfusion des cornes avec $\mathrm{du}$ liquide de Locke, puis placés in vitro, à $37^{\circ} \mathrm{C}$ dans un mélange de $95 \mathrm{p}$. 100 " I 99 " Difco et de 5 p. Ioo de sérum de Lapine, pendant 30 minutes à 2 heures. Le " spéculum " était un tube de verre de $9 \mathrm{~mm}$ de diamètre.

TABLEAU I

Plan de l'expérience 1 .

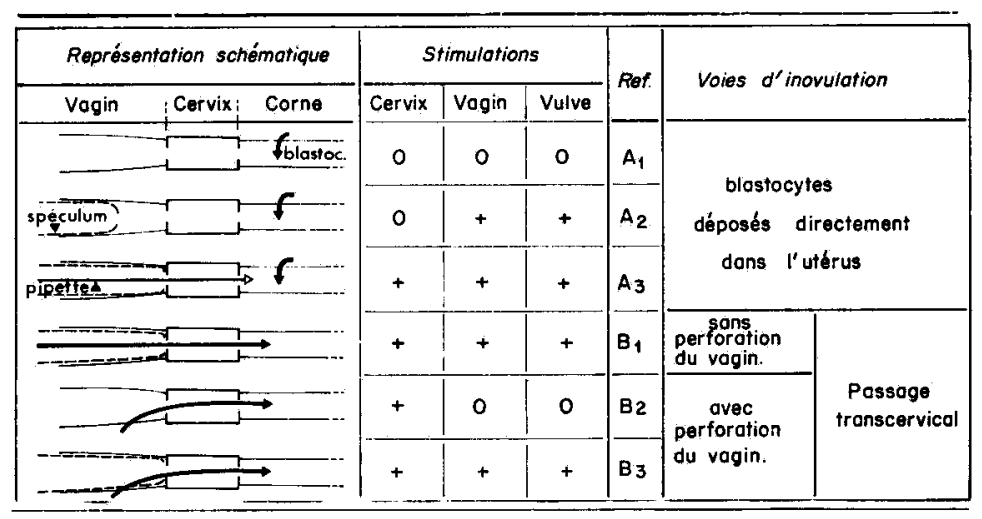

Pour préciser la signification de la différence entre les lots $A_{1}$ et $A_{2}$, une deuxième expérience fut effectuée sur $2 \mathrm{I}$ lapines (Exp. II). Les blastocystes étaient déposés dans la moitié supérieure des cornes utérines et la stimulation mise en jeu dans le lot $\mathrm{A}_{2}^{\prime}$ était modifiée comme suit par rapport à $A_{2}$ : I heure après transfert des blastocystes, un ballonnet sphérique, introduit dans le fond du vagin, était gonflé par Io $\mathrm{ml}$ d'air. Dix déplacements de $13 \mathrm{~cm}$ d'amplitude étaient effectués en I minute selon l'axe céphalo-caudal de l'animal éveillé, puis le ballonnet était extrait gonflé.

Dans ces 2 expériences, les femelles hôtes étaient abattues 7 jours après transfert pour examen de l'utérus. Les sites d'implantation étaient comptés et les embryons de ir jours étaient considérés comme vivants seulement quand le cour battait.

Enfin, pour tester l'importance de l'expulsion éventuelle des œufs hors de l'utérus, un essai fut 
elfectué sur I 5 lapines (Exp. III). Le transfert d'œufs âgés de 2, 3 ou 4 jours dans la moitié supérieure des cornes de femelles 2,3 ou 4 jours p. c. était accompagné d'une ligature du vagin et, dans certains cas, d'une stimulation du cervix (type $\mathrm{B}_{2}$ ). La ligature, placée à $3 \mathrm{~cm}$ du cervix et respectant la vascularisation vaginale, devait permettre la récupération des cufs; ces animaux étaient tués après 24 heures pour rechercher les œufs d'origine (non fécondés) et transplantés (fécondés) dans le liquide de perfusion de l'utérus et du cul-de-sac vaginal.

Au total, 90 transferts ont été effectués.

\section{RÉSULTATS}

Dans certains lots de l'expérience $I$, et plus particulièrement dans ceux qui font intervenir une pénétration du cervix, on trouve à II jours (voir tabl. 2) une proportion importante de lapines montrant des suites opératoires (I 2 sur 39 , soit 3 I p. IOo, avec infection intra-utérine et 9 sur 39 , soit $23 \mathrm{p}$. I00, avec infection musculaire ou adhérences). Il nous a semblé préférable d'analyser 1' " effet stimulation " sur les seuls animaux à tractus normal.

\section{TABLEAU 2}

Exp. 1. - Devenir des lapines après transplantation

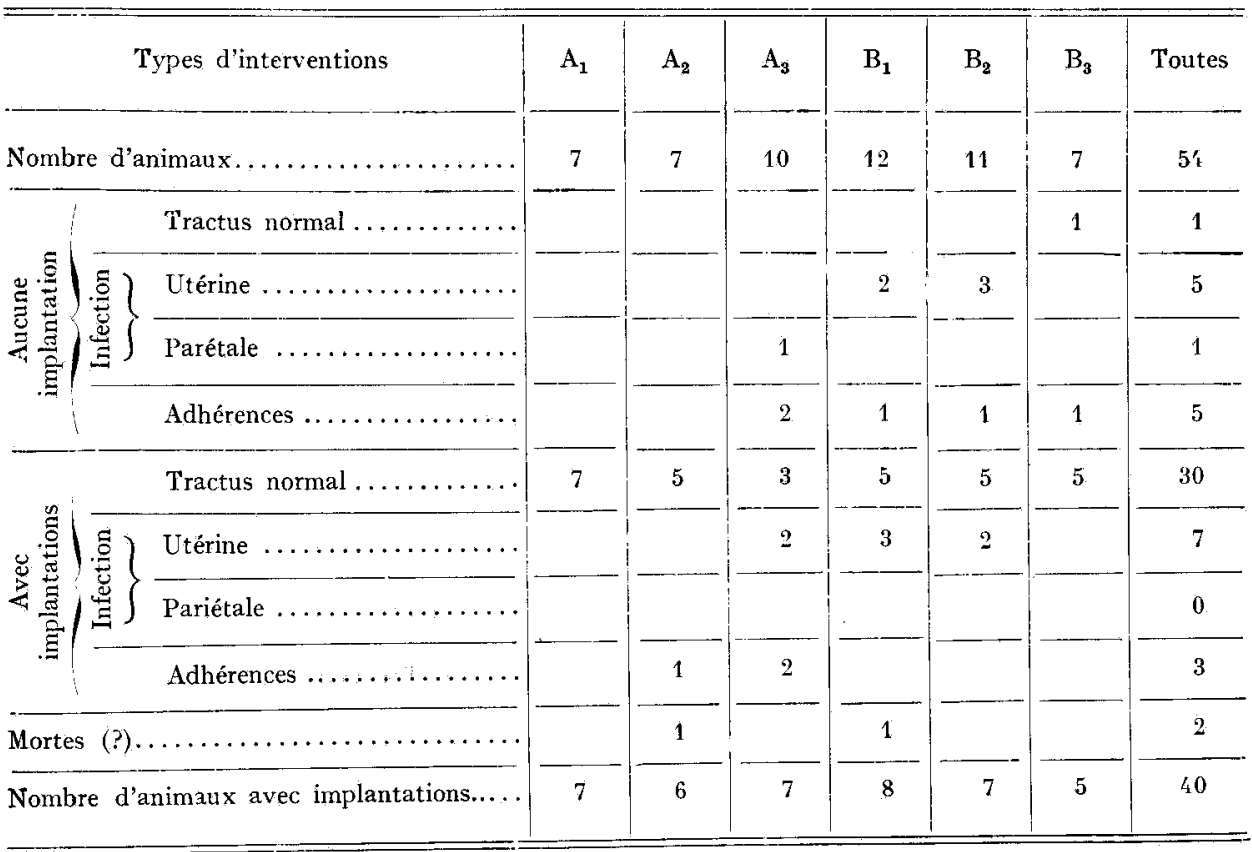

Le tableau 3 montre que si le transfert s'accompagne du passage transcervical d'un cathéter (lots $A_{3}, B_{1}, B_{2}, B_{3}$ ), les taux d'implantations varient de 39 à $5^{\circ} \mathrm{p}$. Ioo $(47,4 ; 50,0 ; 45,3 ; 39$, I respectivement) alors que si le cervix n'est pas franchi, ces taux sont de 70,9 et $8 I, 7$ (lots $A_{1}$ et $A_{2}$ ).

Annales de Biologie animale. - 1969 . 
La différence entre les deux groupes expérimentaux ainsi caractérisés est significative $(\mathrm{P}<0,05)$. Il n'y a pas de différence entre chacun des lots à l'intérieur de ces groupes.

La survie des embryons à II jours, par rapport au nombre d'implantations, ne montre pas de différence entre les 6 lots de lapines.

TABLEAU 3

Exp. I. - Résultats chez les lapines à tractus normal

\begin{tabular}{|c|c|c|c|c|c|c|}
\hline & \multicolumn{6}{|c|}{ Types d'interventions } \\
\hline & $A_{1}$ & $A_{2}$ & $A_{3}$ & $\mathrm{~B}_{1}$ & $\mathrm{~B}_{2}$ & $\mathbf{B}_{\mathbf{3}}$ \\
\hline Nombre d'animaux ........... & 7 & 5 & 3 & 5 & 5 & 6 \\
\hline Nbre de blastocystes transplantés & 93 & 62 & 38 & 62 & 64 & 69 \\
\hline Nombre d'implantations ....... & 76 & 44 & 18 & 31 & 29 & 27 \\
\hline Implantations $(\%) \ldots \ldots \ldots \ldots$ & 81,7 & 70,9 & 47,4 & 50,0 & 45,3 & 39,1 \\
\hline (Intervalle de variation) ........ & $(64-100)$ & $(54-93)$ & $(36-58)$ & $(21-79)$ & $(7-86)$ & $(0-100)$ \\
\hline Nbre d'embryons vivants à $11 \mathrm{j}$ & 70 & 41 & 12 & 28 & 28 & 25 \\
\hline Survie à 11 jours $(\%) \ldots \ldots \ldots$ & 92,2 & 93,2 & 66,7 & 90,3 & 96,5 & 92,6 \\
\hline
\end{tabular}

Les taux d'implantations et de survie des embryons ne sont pas significativement différents dans les lots $A_{2}$ et $A_{1}$, l'expérience II complémentaire $\left(A_{1}\right.$ et $\left.A_{2}^{\prime}\right)$ l'a con firmé : il n'y a donc pas d'influence de la stimulation du vagin entier de l'animal éveillé sur le taux d'implantation ou la survie embryonnaire, à II jours (voir tabl. 4).

\section{TABLEAU 4}

Exp. II. - Influence, sur le rendement, d'une stimulation vaginale 1 heure après la transplantation

\begin{tabular}{|c|c|c|c|c|c|c|}
\hline \multirow[b]{2}{*}{ Types d'interventions } & \multirow[b]{2}{*}{$\begin{array}{l}\text { Nombre } \\
\text { animat } x\end{array}$} & \multirow{2}{*}{$\begin{array}{c}\text { Animaux } \\
\text { à tractus } \\
\text { normal }\end{array}$} & \multirow[b]{2}{*}{ Pyomètre } & \multicolumn{3}{|c|}{ Lapines à tractus normal } \\
\hline & & & & $\begin{array}{l}\text { Nombre de } \\
\text { blastocystes } \\
\text { transplantés }\end{array}$ & $\begin{array}{l}\text { P. } 100 . \\
\text { Implant. } \\
\quad(n)\end{array}$ & $\begin{array}{c}\text { P. } 100 . \\
\text { Survie } \\
11 \text { jours }(n)\end{array}$ \\
\hline$A_{1}$ (témoin-) $\ldots \ldots \ldots \ldots \ldots$ & 11 & 11 & & 118 & $\begin{array}{l}74,6 \\
(88)\end{array}$ & $\begin{array}{l}80,6 \\
(71)\end{array}$ \\
\hline $\begin{array}{l}\mathbf{A}_{2}^{\prime} \\
\text { (Stimulation vaginale) } \ldots \ldots\end{array}$ & 10 & 9 & 1 & 102 & $\begin{array}{l}79,4 \\
(81)\end{array}$ & $\begin{array}{l}83,9 \\
(68)\end{array}$ \\
\hline Total $\ldots \ldots \ldots \ldots \ldots \ldots$ & 21 & 20 & 1 & 220 & $\begin{array}{r}76,8 \\
(169)\end{array}$ & $\begin{array}{l}81,1 \\
(137)\end{array}$ \\
\hline
\end{tabular}




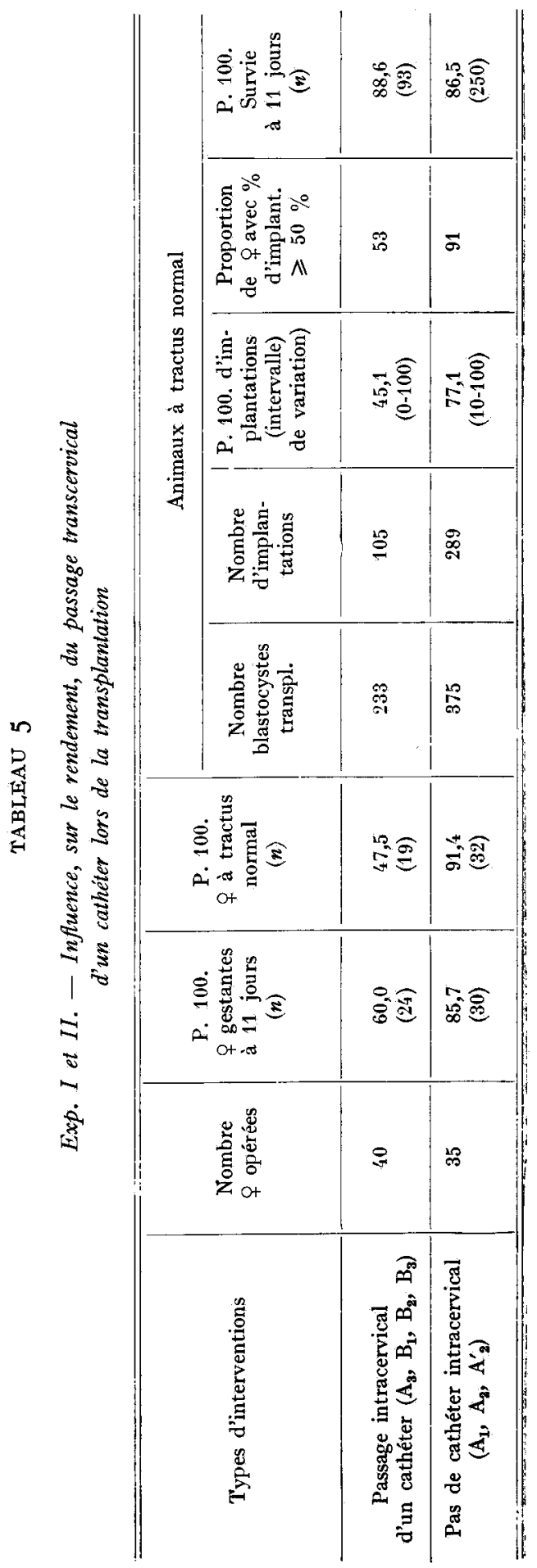


En effet, on trouve 79,4 p. Ioo d'implantations et 83,9 p. Ioo de survie chez les Lapines subissant une telle stimulation contre 74,6 p. Ioo d'implantations et 80,6 p. Ioo de survie chez les témoins.

L'absence de différence $(P<0,05)$ entre les lots $A_{1}, A_{2}$ et $A^{\prime}{ }_{2}$ d'une part, $A_{3}, B_{1}$, $B_{2}$ et $B_{3}$ d'autre part, nous permet de regrouper les résultats de ces lots en 2 groupes ne se différenciant que par un passage transcervical d'un cathéter (tabl. 5).

On constate que diffèrent :

- le taux d'implantation (77,I contre 45,I ; $P<0,01$ ) ;

- la proportion de femelles à tractus normal ( $9 \mathrm{I}, 4$ contre $47.5 ; \mathrm{P}<0, \mathrm{OI}$ )

- la proportion de femelles gestantes $(85,7$ contre $60 \mathrm{p}$. I00; $\mathbf{P}<0,05)$.

Par contre, quand l'implantation s'est produite, les taux de survie à II jours sont semblables dans les deux groupes $(86,5$ et 88,6$)$.

L'expérience III montre que le transfert à 3 ou 4 jours post coitum, associé ou non au passage transcervical d'un cathéter, n'est pas suivi durant les premières 24 heures de l'expulsion des oufs dans le vagin.

Sur I38 blastocystes transférés dans 9 lapines, aucun n'est retrouvé dans le vagin, et 118 , soit 85 p. Ioo, sont récupérés dans l'utérus chez roo p. Ioo des animaux (tab1. 6).

Dans le cas de transfert à 2 jours post coitum, 2I sur I03 blastocystes transférés sont retrouvés dans le vagin de 4 sur 6 lapines et 36 , soit 35 p. Ioo, dans l'utérus chez roo p. roo des animaux.

Dans aucun cas on n'a retrouvé dans le vagin des œufs de la lapine receveuse (œufs d' " origine ").

\section{DISCUSSION}

Io Pour HAFEz (I962), le passage transcervical du cathéter d'inovulation n'affecte pas la réussite de transferts de morulas de lapine de 3 jours. Nous ne retrouvons pas ce résultat en transplantant des blastocystes de 4 jours. L'augmentation, seulement à partir $\mathrm{du} 3^{\mathrm{e}}$ jour, du taux sanguin de progestines (20 $\alpha$-progestérol et progestérone) chez la Lapine (Hil,iaRd, SpIES et SAWYER, I968) permet d'envisager une explication possible : 1'expulsion, dans le vagin, des œufs ainsi transplantés, conséquence de la contraction utérine induite par la stimulation du cervix chez l'animal en phase progestative.

Mais, l'expérience III montre (tab1. 6) que le transfert effectué à partir du troisième jour post-coitum n'est pas suivi de l'expulsion des œufs hors de l'utérus, ce qui confirme le résultat de DAUzIER (I962) puisque, chez des lapines traitées comme celles de notre lot $B_{1}$, il retrouvait encore 52 p. Ioo des blastocystes 6 à 8 heures après transplantation.

Si la Vache répond aux stimulations du tractus génital par une décharge dans l'organisme d'une substance à action ocytocique (HAYS et VANDEMARK, I953) susceptible de provoquer l'expulsion dans le vagin de particules placées in utero (Rowson, BENNETT et HARPER, I964; TestaRT, non publié), la Lapine, dont le muscle utérin en phase progestative montre une insensibilité totale in vitro à 1'hormone posthypophysaire (KNAUS, I93I), ne présente pas la même réaction. 


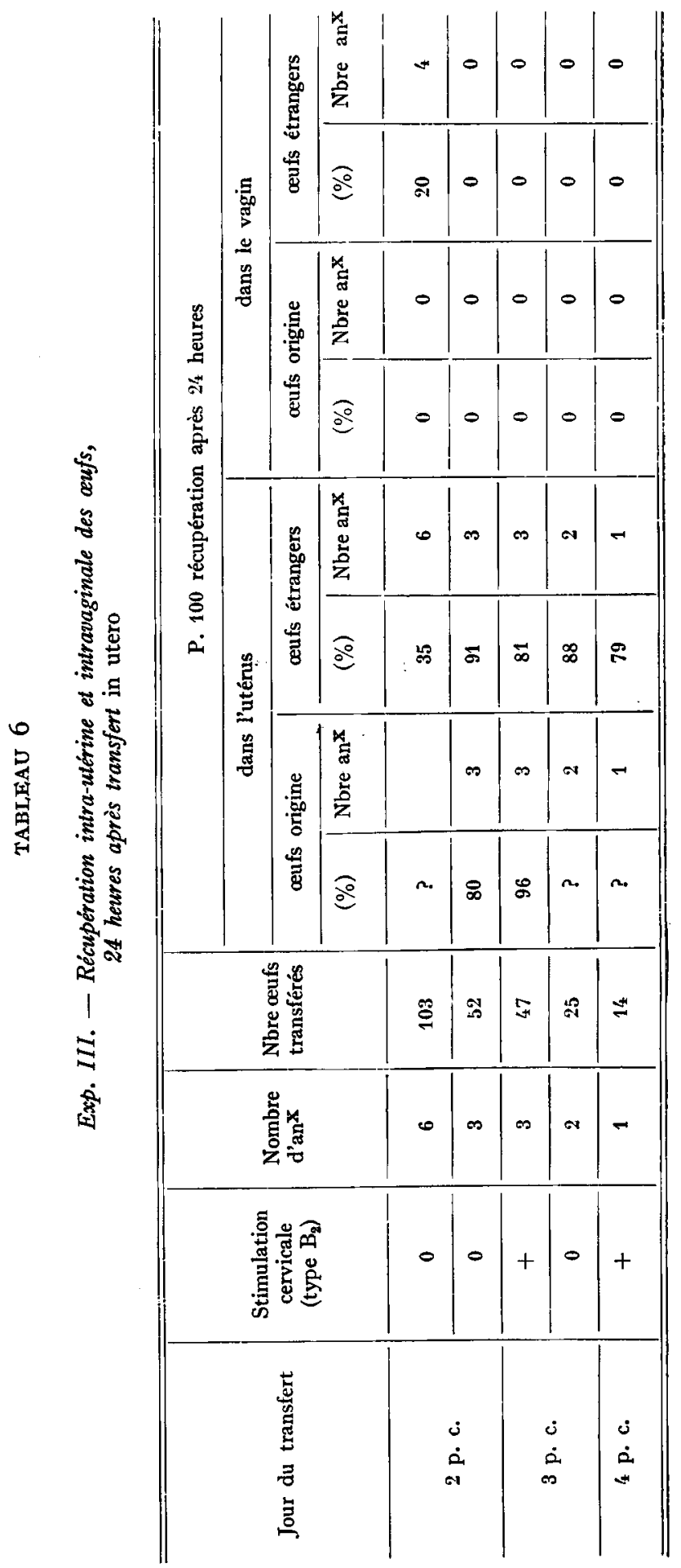


Aussi sommes-nous amenés à éliminer le facteur " expulsion " dans l'explication de nos résultats.

$2^{\circ}$ L'absence de différence $(P<0,05)$ entre les rendements des lots faisant intervenir le passage transcervical d'un cathéter, avec ou sans introduction vaginale du spéculum, montre que les stimulations vulvaires et vaginales sont sans effet sur ce rendement. L'expérience II confirme ce résultat. Seul le passage intracervical du cathéter entraîne une différence entre les lots expérimentaux pour les taux d'implantations, les proportions de femelles à tractus normal et de femelles gestantes. Sans éliminer l'influence possible de cette manipulation sur l'activité ovarienne, la proportion anormalement basse de femelles à tractus normal $(47,5 \mathrm{p}$. Ioo contre $9 \mathrm{I}, 4 \mathrm{p}$. IOo) nous amène à donner une importance prépondérante aux réactions inflammatoire et traumatique (adhérences que nous attribuons à la manipulation prolongée de l'utérus).

$3^{\circ}$ La réaction inflammatoire, sous forme de pyomètre, est présente chez $30 \mathrm{p}$. roo des animaux ( 12 sur 40, tab1. 2) ayant subi le passage transcervical d'un cathéter au $4^{\mathrm{e}}$ jour de pseudogestation. Cependant, si on ne considère que les femelles dont le tractus est d'apparence normale à II jours, seulement $45 \mathrm{p}$. Ioo des blastocystes se sont implantés contre $77 \mathrm{p}$. roo chez les femelles ayant subi un transfert direct dans 1'utérus.

Les variations inter-individuelles observées dans les rendements soutiennent l'hypothèse d'une réaction infectieuse d'intensité variable selon la qualité de l'intervention et l'importance de la défense de l'organisme.

9I $\mathrm{p}$. roo des lapines des lots $A_{1}, A_{2}$ et $A_{2}^{\prime}$ montrent au moins $50 \mathrm{p}$. Ioo d'implantations contre $53 \mathrm{p}$. Ioo de celles des lots $A_{1}, B_{2}, B_{2}$ et $B_{3}$ (différence significative, $\mathbf{P}<0,0 I)$ bien que les intervalles des variations individuelles soient sensiblement identiques (ro à roo $\mathrm{p}$. Ioo pour le $\mathrm{I}^{\mathrm{er}}$ groupe, contre o à Ioo $\mathrm{p}$. Ioo pour le second) (tabl. 5).

La réaction infectieuse, lors d'une contamination bactérienne de l'utérus en phase lutéale, a été vérifiée dans plusieurs espèces. Chez la Vache (Rowson, LAMMING et FRY, I953 ; DzIUK et al., I958), la Brebis (BRINSFIELD, HAwK et LEFFEI, I967) et la Lapine (Black et al., I953 ; BRoOmE et LAMming, I958 ; BRoOME, LAMMING et SMITH, I959), on connaît la plus grande résistance à l'infection utérine de la femelle en cstrus et, suivant les auteurs, les hormones ovariennes joueraient un rôle déterminant dans cette résistance, en agissant soit sur le nombre et la vitesse de mobilisation des leucocytes, soit sur la capacité antibactérienne de ces leucocytes, soit sur le drainage de l'utérus.

Le moment où nous pratiquons la transplantation des blastocystes ( $4^{\mathrm{e}}$ jour) se situe en phase d'augmentation du taux de progestines circulantes (HII,IARD, SPIES et SAWYer, I968), or Lalmming et Heap (I960), chez la Lapine pseudo-gestante, envisagent dans la fraction acido-soluble d'extraits utérins, la présence d'une substance qui, sous dominance progestéronique, empêcherait 1'infiltration leucocytaire. Ces mêmes auteurs distinguent deux aspects du mécanisme de défense : 1'infiltration et 1'activité leucocytaire ; KIL,INGBECK et LAMMING (I963) ont trouvé dans les sécrétions utérines de la Lapine en phase lutéale un séro-mucoïde qui inhiberait l'activité des leucocytes.

$4^{0}$ Nous devons remarquer que le résultat du lot $B_{1}$, ne diffère pas sensiblement 
de celui de HAFEZ par la même voie ( 50 p. Ioo et 59 p. I00) ; ils sont tous deux supérieurs au rendement obtenu par DAUZIER ( 7 p p IOO). De même, le résultat de notre lot $B_{2}$, ne diffère pas sensiblement de celui de DAUZIER (45,3 p. Ioo et $47 \mathrm{p}$. IOo). Notre pourcentage plus élevé que celui de HAFEz par voie transutérine $(8 \mathrm{r}, 7 \mathrm{p}$. Ioo contre 54 à $60 \mathrm{p}$. Ioo), obtenu à plusieurs reprises, à des moments différents de l'année sur des groupes d'animaux différents, peut s'expliquer par une résistance supérieure des blastocystes de 4 jours, un meilleur état sanitaire des animaux ou le rôle de détails méthodologiques liés à la réaction inflammatoire.

Reç pour publication en mars 1969.

\title{
SUMMARY
}

\author{
INVESTIGATIONS ON VARIOUS WAYS OF TRANSPLANTATION \\ OF BLASTOCYSTS IN THE RABBIT
}

75 transplantations of 4 day-blastocysts ( 4 to 7 blastocysts per uterine horn) were conducted on female rabbits. The vulva, vagina or cervix were stimulated according to the different pathways of transfer (table $\mathrm{r}$ ).

Transfers conducted by directly depositing the blastocysts in the uterus $\left(\mathrm{A}_{1}, \mathrm{~A}_{2}, \mathrm{~A}_{2}^{\prime}\right.$ experiments) resulted in 85.7 per cent of gravid females; 77.1 implantations and 86.5 fetal survivals at day II of pregnancy per roo uninfected females. (table 5). Mechanical stimulation of the vagina at the time of transfer $\left(A_{2}\right)$ or one hour after anaesthetization $\left(A_{2}{ }^{\prime}\right)$ had no influenfce on the results. (Tables 3 and 4 ).

Transfers conducted via the cervix by means of an inovulation catheter $\left(B_{1}, B_{2}, B_{3}\right.$, experiments), and direct transfer accompanied by the passage of an inovulation catheter through the cervix ( $A_{3}$ experiment), resulted in 60 per cent of gravid females, 45.1 implantations and 88.6 fetal survivals at day II of pregnancy per ioo uninfected females (Table 5).

There were significant differences between the two modes of transfer, in the rates of gravid females $(P<0.05$ ) and implantations $(P<0.0 I)$; however, the fetal survivals of implanted blastocysts were similar.

At day II of pregnancy, 30 per cent of hosts inovulated via the cervival pathway showed pyometritis (table 2), and only 47.5 per cent had normal tracts, versus 9r.4 per cent whith direct transfer. This difference is highly significant.

3- or 4-day transfers were not followed by the expulsion of ova, whereas 24 hours after 2day direct transfer, 20 per cent of ova were found in the vagina of 67 per cent of females (table 6).

The passage through the septic medium of the vagina and the lower resistance of the uterus to bacterial infection during the progestational stage accounts for the greater inadequacy of the cervical pathway of inovulation.

\section{RÉFÉRENCES BIBLIOGRAPHIQUES}

Avery T. L., Fahning M. L., Pursel V. G., Graham E. F., ig62. Investigations associated with the transplantation of bovine ova. IV . Transplantation of ova. J.Reprod. Fert., 3, 229-238.

BEATty R. A., I95I. Transplantation of mouse eggs. Nature, 168, 995.

Black W. G., Simon J., McNuTT S. H., CASIDA L. E., 1953. Investigation on the physiological basis for the differential response of estrous and pseudopregnant rabbit uteri to induced infection. $A$ mer. $J$. Vet. Res., 14, 318-323.

Brinsfield . T. H., Hawk H. W., LefFel E. C., I967. Control by ovarian status of induced leukocytic responses in the sheep uterus. Amer. J. Vet. Res., 28, 127.

Broome A. W. J., LAmming G. E., I958. The influence of ovarian hormones on resistance to uterine infections. Vet. Rec., 70, 603-604. 
Broome A. W. J., Lamming G. E., Smith W., 1959. Studies on the relationship between ovarian hormones and uterine infection. IV. The role of the granulocyte system in uterine defence. $J$. Endrocr., 18, 274-28 I.

Dauzier L., I962. Nouvelles données sur la transplantation des oufs chez la Lapine, par voie vaginale ou intrapéritonéale. Ann. Biol. anim. Bioch. Biophys., 2, I7-24.

Dowling D. F., I949. Problems of the transplantation of fertilized ova. J. Agric. Sci., 39, 374*396.

Dziuk P. J., Donker J. D., Nichols J. R., Petersen W. E., 1958. Problems associated with the transfer of ova between cattle. Univ. Minnesota Agric. Exp. Sta. Tech. Bull., 222, 75 pp.

HaFE2 E. S. E., I962. Effect of progestationnal stage of the endometrium on implantation, fetal survival and fetal size in the rabbit. J. Exp. Zool., 151, 2 I 7-225.

Hafez E. S. E., Sugie T., 1963. Reciprocal transfer of cattle and rabbit embryos. J. Anim. Sci., 22,

HAYS R. L., VANDEMaRK N. L., r953. Effect of stimulation of the reproductive organs of the cow on the release of an oxytocin-like substance. Endocrinology, 52, 634-637.

Hilliard J., Spies H. G., SAwYer C. H., I968. Cholesterol storage and progestin secretion during pregnancy and pseudopregnancy in the rabbit. Endocrinology, 82, $157-165$.

Killingbeck J., LAmming G. E., I963. Influence of uterine secretions on phagocytosis. Nature, London, 198, III-II2.

KNaUs H., I931. Der biologische Test für das Corpus luleum-Hormon. Klinische Wochenschr., 10, 743.

Lamming G. E., r96r. The influence of ovarian hormones on resistance to uterine infection (Proc.). $J$. Reprod. Fert., 2, 517-518.

LAMming G. E., RowSON L. E. A., 1952. Superovulation and ovum transplantation in cattle. IIe Int. Congr. Physiol. Pathol. Anim. Reprod. Artif. Insem., Copenhagen, 1, I44-153-

Lamming G. E., Heap R. G., I960. Studies of the uterine environment of different species. II. The influence of ovarian hormones on experimental uterine infections. J. Endocr., 20, XXIV.XXV (Proc.)

Mutter L. R., Graden A. P., Olds D., 1965. Successful non-surgical bovine embryo transfer. $A$. 1 . Digest, 12 (I I), 3 .

OTSUKI K., Soma T., I964. Transfer of fertilized ova through the cervix in goats. Bull. Nal. Inst . Anim. Ind., 6, 27-32.

Rowson L. E. A., Lamming G. E., Fry R. M., 1953. The relationship between ovarian hormones and uterine infection. Vet. Rec., 65, 335-343.

Rowson L. E. A., Bennett J. P., Harper M. J. K., i964. The problem of non-surgical egg transfer to the cow uterus. Vet. Rec., 76, $2 \mathrm{I}-23$.

Rowson L. E. A., Moor R. M., I966. Non-surgical transfer of cow eggs. J. Reprod. Fert., 11, 311-312.

Sugie. T., 1965. Successful transfer of a fertilized bovine egg by non-surgical technique. J. Reprod. Ferl,, $10,197-202$. 